\title{
The influence of AGN nuclear parameters on the FRI/FRII dichotomy
}

\author{
M. Wold ${ }^{1,2,3}$, M. Lacy ${ }^{2}$, and L. Armus ${ }^{2}$ \\ ${ }^{1}$ European Southern Observatory, Karl-Schwarzschild str. 2, 85748 Garching bei München, Germany \\ e-mail: wold@astro.uio.org \\ 2 Spitzer Science Center, California Institute of Technology, MS 220-6, Pasadena, CA 91125, USA \\ 3 Present address: Institute of Theoretical Astrophysics, PO Box 1029 Blindern, 0315 Oslo, Norway
}

Received 24 March 2006 / Accepted 4 May 2007

\begin{abstract}
We have investigated the influence of nuclear parameters such as black hole mass and photoionizing luminosity on the FRI/FRII transition in a sample of nearby $(z<0.2)$ radio galaxies from the 3CR catalogue. The sample was observed with medium-resolution, optical spectroscopy and contains some galaxies with unpublished velocity dispersion measurements and emission-line fluxes. The measured velocity dispersions for the sample lie in the range $130-340 \mathrm{~km} \mathrm{~s}^{-1}$ with a mean of $216 \mathrm{~km} \mathrm{~s}^{-1}$. Using the M- $\sigma_{*}$ relation, we convert to black hole mass and find that the black hole mass distribution is identical for FRI and FRII galaxies, with a mean of $\approx 2.5 \times 10^{8} M_{\odot}$. We determine narrow emission-line luminosities from [O II] and [O III] in our spectra, as well as from the literature, and convert them to photoionizing luminosities under the assumption that the gas is ionized by the nuclear UV continuum. Most of the galaxies with FRI morphology and/or low-excitation emission-line spectra have progressively lower black hole masses at lower photoionizing (and jet) luminosities. This agrees with the well-known Ledlow-Owen relation which states that the radio luminosity at the FRI/FRII transition depends on the optical luminosity of the host, $L_{\text {radio }} \propto L_{\text {optical }}^{1.8}$, because these two luminosities relate to AGN nuclear parameters. When recasting the Ledlow-Owen relation into black hole mass versus photoionizing luminosity and jet luminosity, we find that the recasted relation describes the sample quite well. Furthermore, the FRI/FRII transition is seen to occur at approximately an order of magnitude lower luminosity relative to the Eddington luminosity than the soft-to-hard transition in X-ray binaries. This difference is also consistent with the Ledlow-Owen relation, which predicts a weak black hole mass dependence in the transition luminosity in Eddington units. We conclude that the FRI/FRII dichotomy is caused by a combination of external and nuclear factors, with the latter dominating.
\end{abstract}

Key words. galaxies: active - galaxies: nuclei - galaxies: jets - X-rays: binaries

\section{Introduction}

Radio galaxies are usually classifed as FRI or FRII sources depending on their radio morphology. FRIs have smooth jets emanating from the nucleus and lobes where the surface brightness decreases toward the edges. The more powerful FRIIs have faint jets and edge-brightened lobes. The classification scheme was invented by Fanaroff \& Riley (1974), who discovered that the FRI/FRII transition occurs at a radio luminosity of $P_{178 \mathrm{MHz}} \approx$ $10^{25} \mathrm{~W} \mathrm{~Hz}^{-1} \mathrm{sr}^{-1}$, with almost all sources below the transition luminosity being FRIs.

Over the years, much work has been put into understanding the remarkable FRI/FRII transition. Models fall into two different groups, those explaining the morphological differences as arising because of different physical conditions in the environment in which the radio source propagates, and those seeking to explain the dichotomy as caused by fundamental AGN parameters or the jets (see Gopal-Krishna \& Wiita 2000, for a summary). The deceleration models, belonging to the former group, have been successful in reproducing the appearance of both FRI and FRII jets. In this scenario, the jets are thought to start out supersonically and slow down to a tran/subsonic flow because of entrainment of plasma in the host galaxy (De Young 1993; Laing 1994, 1996; Bicknell 1995; Kaiser \& Alexander 1997). The other group of models explains the differences as arising in more fundamental parameters like black hole $(\mathrm{BH})$ spin, accretion mode, or jet composition (Rees et al. 1982; Baum et al. 1995; Reynolds et al. 1996; Meier 1999, 2001; Ghisellini \& Celotti 2001; Marchesini et al. 2004).

An interesting discovery was made by Ledlow \& Owen (1996) (see also Owen \& Laing 1989; Owen \& White 1991; Owen \& Ledlow 1994) who found that the FRI/FRII transition radio luminosity is an increasing function of the optical luminosity of the host galaxy, $L_{\mathrm{rad}} \propto L_{\mathrm{opt}}^{2}$. This means that the more optically luminous a galaxy is, the more powerful its radio source must be in order to produce FRII morphology. The LedlowOwen relation has been an important observation in the effort to explain radio galaxy dichotomy. However, both groups of models are able to explain, or reproduce, the Ledlow-Owen relation (Bicknell 1995; Meier 1999; Gopal-Krishna \& Wiita 2001).

From the correlation between bulge luminosity and BH mass (Kormendy \& Richstone 1995; Magorrian et al. 1998), we expect the host optical luminosity to scale with $\mathrm{BH}$ mass. Furthermore, because the radio-optical correlation (optical here means nuclear optical luminosity) for radio galaxies (Saunders et al. 1989; Rawlings \& Saunders 1991; Willott et al. 1999; Grimes et al. 2004) relates radio luminosity to narrow-line luminosity, the radio luminosity can be expressed as a function of nuclear photoionizing luminosity. The Ledlow-Owen relation can thus be cast into the variables BH mass and nuclear photoionizing luminosity. Ghisellini \& Celotti (2001) use this line 
of reasoning to argue that the Ledlow-Owen relation reflects a change in the accretion mode between the two classes because the transition occurs at a fixed ratio between $\mathrm{BH}$ mass and photoionizing/accretion luminosity. Recent work by Marchesini et al. (2004) also argues for a change in the accretion mode. They study a sample of radio-loud quasars and radio galaxies, finding that whereas quasars and radio galaxies have a similar distribution in $\mathrm{BH}$ mass, the distribution of accretion rates is bimodal with FRIs and weak-lined FRIIs on one side, and FRIIs and radio-loud quasars on the other.

In the above-mentioned work, optical luminosity is used as a measure of photoionizing luminosity. It is however still a matter of controversy whether this is applicable to FRI galaxies because it is uncertain whether they have dusty torii that can obscure the nucleus, leading to an underestimate of their true optical luminosity. One way to avoid the ambiguity in the interpretation of optical (and X-ray) nuclear luminosity is to use narrow emission lines as these are believed to arise from the narrow-line region outside the torus and be photoionized by the UV continuum from the central AGN.

In this paper we revisit the Ledlow-Owen relation for a sample of nearby FRI and FRII galaxies by utilizing stellar velocity dispersions to estimate $\mathrm{BH}$ masses and narrow emission-line luminosities to estimate photoionizing luminosities. We assume a cosmology with $H_{0}=70 \mathrm{~km} \mathrm{~s}^{-1} \mathrm{Mpc}^{-1}, \Omega_{\mathrm{m}}=0.3$ and $\Omega_{\Lambda}=0.7$ unless otherwise noted. The abbreviations [O II] and [O III] are used for [O II] $3727 \AA$ and [O III]5007 throughout the paper.

\section{The sample}

The sample consists of sources drawn from the complete 3CRR catalogue of Laing et al. (1983). The selection criterion was $P_{178} \leq 10^{26.5} \mathrm{~W} \mathrm{~Hz}^{-1} \mathrm{sr}^{-1}$, hence covering the FRI/FRII transition regime. Further constraints on RA and Dec gave a sample of 21 targets, ten FRIs and eleven FRIIs. Because of the redshift-luminosity correlation in the 3CRR sample, all targets lie at $z<0.2$. The properties of the selected sources are listed in Table 1. Radio power at rest-frame $178 \mathrm{MHz}$ was calculated from the $178 \mathrm{MHz}$ flux and the spectral index as listed in the 3 CRR Atlas web pages by Leahy, Bridle and Strom ${ }^{1}$. Radio morphology class was taken from the same web pages. In addition to morphology, we also list emission-line class as either high-excitation ("HEG") or low-excitation ("LEG") emission line galaxy. FRIIs are classified as HEGs if [O III] is strong compared to [O II], and as LEGs if [O III] is weak or absent (Laing et al. 1994). FRIs have low-excitation spectra, hence all the FRIs are listed as LEGs in Table 1.

Emission-line classes for all objects were found in the literature (see table note) except for 3C 442A, which we classify as a LEG because [O III] is weaker than [O II] in our spectrum. This galaxy is not a classical FRII with well-defined hot spots (one of the lobes has FRI morphology), and the host galaxy is part of an ongoing merger (Comins \& Owen 1991). The other source with non-classical radio morphology in the sample is 3C 433, highly asymmetric and probably interacting with close neighbours. The radio morphology is classified as FRI by Leahy, Bridle and Strom, but we have chosen to define it as an FRII because its optical spectrum is characteristic of an HEG and there is evidence that it harbours an obscured quasar nucleus (Fabbiano et al. 1984; Lilly et al. 1985; Yates \& Longair 1989).

\footnotetext{
${ }^{1}$ http://www.jb.man.ac.uk/atlas
}

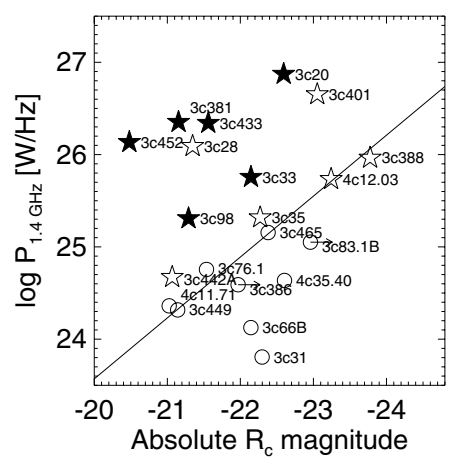

Fig. 1. Open circles correspond to FRIs and stars to FRIIs, with filled symbols for high-excitation emission line galaxies. The solid line marks the Ledlow-Owen relation.

In order to compare the sources with the Ledlow-Owen relation, we plot the sample in the radio-optical plane in Fig. 1. The apparent rest-frame $R_{\mathrm{c}}$ magnitudes were converted to absolute magnitudes, and radio luminosity at $1.4 \mathrm{GHz}$ calculated using the observed flux at $1.4 \mathrm{GHz}$ and spectral indices as listed in Table 1. For calculating luminosities, we used the same cosmology as Ledlow \& Owen (1996). The figure shows that the galaxies fall in the expected regions of the diagram, with FRI sources below and FRII sources above the Ledlow-Owen relation, but there are also some borderline cases. All galaxies classified as HEGs are well above the relation, consistent with the unified schemes (Jackson \& Wall 1999; Chiaberge et al. 2002).

\section{Observations and data reduction}

Medium resolution ( $R \sim 3000-4000)$ optical spectra were obtained with the Double Spectrograph (Oke \& Gunn 1982) on the $5 \mathrm{~m}$ Hale Telescope at Palomar Observatory. The spectrograph was equipped with two $1 \mathrm{k} \times 1 \mathrm{k}$ CCDs and the D48 dichroic, dividing the red and blue side at approximately $4800 \AA$. We utilized the $1200 \mathrm{l} / \mathrm{mm}$ grating blazed to $7100 \AA$ on the red CCD and to $4700 \AA$ on the blue CCD. Two different grating angles were used depending on the galaxy redshift such that the Mg I $b \lambda 5175 \AA$ absorption line complex fell on the center of the red CCD, and the $\mathrm{Ca} \mathrm{H} \& \mathrm{~K} \lambda \lambda 3934,3969 \AA$ doublet at the center of the blue CCD. The red and the blue CCDs had pixel scales of $0 . ' 468$ and 0 ' $^{\prime} 624$, resulting in dispersions of 0.624 and $0.864 \AA /$ pix, respectively.

Absorption line spectra were obtained with a $1^{\prime \prime}$ wide slit, giving spectral resolutions of 1.33 and $1.39 \AA$, corresponding to velocity resolutions of $\approx 70$ and $\approx 100 \mathrm{~km} \mathrm{~s}^{-1}$ in the red and the blue, respectively. In order to measure total narrow emission line fluxes, we also took spectra with a wide slit $\left(4^{\prime \prime}\right)$ of part of the sample. Slit lengths were $128^{\prime \prime}$. Spectra were taken by centering the slit on the optical nucleus of the galaxy and aligning it with the major axis in order to maximize the amount of light entering the slit. Each target was also observed as close to the parallactic angle as possible in order to minimize losses due to differential atmospheric refraction. Details about the observations are given in Table 2.

Wavelength calibration was accomplished by taking arc lamp spectra at every telescope pointing and standard stars were observed at the beginning and end of each night. There were some clouds at the beginning of the first night, but for the rest of the run the weather was clear and photometric, and the seeing stable at $\approx 1^{\prime \prime}$. The photometric zero point varied $0.1-0.2 \mathrm{mag}$ 
Table 1. The sample. $P_{178}$ in Col. (3) is total radio power $\left(\mathrm{W} \mathrm{Hz}^{-1} \mathrm{sr}^{-1}\right)$ at rest-frame $178 \mathrm{MHz}$. Column (4) lists spectral index between 178 and $750 \mathrm{MHz}$, and Cols. (5) and (6) the radio morphology and emission-line type. Apparent magnitude in rest-frame $R_{\mathrm{C}}$-filter and corresponding references are listed in Cols. (7) and (8). Columns (9) and (10) show velocity dispersion measurements from the literature (km s${ }^{-1}$ ) and corresponding references. The last three columns list emission line fluxes in units of $10^{-15} \mathrm{erg} \mathrm{s}^{-1} \mathrm{~cm}^{-2}$, both from the literature and as determined from our wide slit spectrophotometry, and references.

\begin{tabular}{|c|c|c|c|c|c|c|c|c|c|c|c|c|}
\hline $\begin{array}{l}\text { Radio ID } \\
\text { (1) }\end{array}$ & $\begin{array}{l}\text { Redshift } \\
\text { (2) }\end{array}$ & $\begin{array}{l}\log P_{178} \\
\text { (3) }\end{array}$ & $\begin{array}{l}\alpha_{178}^{750} \\
(4)\end{array}$ & (5) & (6) & $\begin{array}{l}m_{R_{\mathrm{C}}} \\
(7)\end{array}$ & $\begin{array}{l}\text { Ref. } \\
\text { (8) }\end{array}$ & $\begin{array}{l}\sigma_{\text {lit }} \\
(9)\end{array}$ & $\begin{array}{l}\text { Ref. } \\
\text { (10) }\end{array}$ & $\begin{array}{l}S_{\text {[OII] }} \\
(11)\end{array}$ & $\begin{array}{l}S_{\text {[OIII] }} \\
(12)\end{array}$ & $\begin{array}{l}\text { Ref. } \\
\text { (13) }\end{array}$ \\
\hline $3 \mathrm{C} 20$ & 0.174 & 26.49 & 0.66 & FRII & HEG & 16.80 & 1 & & & 0.634 & 1.915 & $\mathrm{~W}$ \\
\hline $3 C 28$ & 0.1971 & 26.22 & 1.06 & FRII & LEG & 18.34 & 2 & & & 5.956 & $\ldots$ & W \\
\hline $3 \mathrm{C} 31$ & 0.0167 & 23.99 & 0.57 & FRI & LEG & 11.92 & 2 & 249 & SHI90 & .. & 10.349 & G \\
\hline $3 \mathrm{C} 33$ & 0.0595 & 25.60 & 0.76 & FRII & HEG & 14.80 & 3 & & & 33.017 & 147.48 & W \\
\hline 3 C 35 & 0.0673 & 24.99 & 0.77 & FRII & LEG & 14.94 & 1 & & & $1.0 \pm 0.2$ & $<0.34$ & A \\
\hline $3 \mathrm{C} 66 \mathrm{~B}$ & 0.0215 & 24.35 & 0.50 & FRI & LEG & 12.55 & 2 & & & 6.782 & 4.801 & W \\
\hline 3C 76.1 & 0.0324 & 24.41 & 0.77 & FRI & LEG & 14.06 & 2 & 246 & SHI90 & $<3.0$ & $<2.0$ & A \\
\hline $3 \mathrm{C} 83.1 \mathrm{~B}$ & 0.0255 & 24.53 & 0.62 & FRI & LEG & $<12.11$ & 4 & & & & & \\
\hline 3С 98 & 0.0306 & 24.95 & 0.78 & FRII & HEG & 14.18 & 3 & 175 & SHI90 & 7.936 & 39.775 & W \\
\hline 3 C 381 & 0.1605 & 26.01 & 0.81 & FRII & HEG & 18.05 & 5 & & & 1.177 & 44.31 & W \\
\hline 3C 386 & 0.0170 & 24.13 & 0.59 & FRI & LEG & $<12.21$ & 4 & & & & & \\
\hline 3 C 388 & 0.0908 & 25.64 & 0.70 & FRII & LEG & 14.12 & 3 & 365 & H85 & 1.618 & 1.901 & W \\
\hline $3 \mathrm{C} 401$ & 0.201 & 26.32 & 0.71 & FRII & LEG & 16.68 & 6 & & & $\ldots$ & 1.192 & W \\
\hline $3 \mathrm{C} 433$ & 0.1016 & 26.10 & 0.75 & FRII & HEG & 16.59 & 5 & & & $\ldots$ & 2.656 & W \\
\hline $3 \mathrm{C} 442 \mathrm{~A}$ & 0.0263 & 24.35 & 0.96 & FRII & LEG & 14.07 & 2 & 197 & SHI90 & $8.0 \pm 0.8$ & $4.7 \pm 1.0$ & A \\
\hline 3C 449 & 0.0171 & 23.82 & 0.58 & FRI & LEG & 13.05 & 2 & 222 & SHI90 & $<3.0$ & $<2.0$ & A \\
\hline $3 \mathrm{C} 452$ & 0.0811 & 25.88 & 0.78 & FRII & HEG & 17.16 & 7 & & & 17.149 & $\ldots$ & W \\
\hline $3 \mathrm{C} 465$ & 0.0305 & 24.87 & 0.75 & FRI & LEG & 13.14 & 2 & 341 & FIF95 & $2.0 \pm 0.4$ & $<4.0$ & A \\
\hline 4C 11.71 & 0.0262 & 24.12 & 0.75 & FRI & LEG & 14.00 & 7 & 249 & EFAR99 & $<5.0$ & $<3.0$ & A \\
\hline 4C 12.03 & 0.157 & 25.77 & 0.87 & FRII & LEG & 15.91 & 1 & & & $\ldots$ & 1.394 & W \\
\hline 4C 35.40 & 0.0301 & 24.29 & 0.76 & FRI & LEG & 12.83 & 8 & 204 & EFAR99 & $1.0 \pm 0.2$ & $<1.3$ & A \\
\hline
\end{tabular}

References for $m_{R_{\mathrm{C}}}$ : (1) Converted from $K_{\mathrm{UKIRT}}$ (Lilly \& Longair 1984) assuming $R_{\mathrm{C}}-K=2.5$ (Dunlop et al. 2003); (2) Cao \& Rawlings (2004); (3) Owen \& Laing (1989); (4) Martel et al. (1999) (upper limit given because of contamination by foreground star); (5) converted from $V$ (Smith \& Heckman 1989) assuming $V-R_{\mathrm{C}}=0.61$ (Fukugita et al. 1995); (6) converted from $K$ (Lebofsky 1981) assuming $R_{\mathrm{C}}-K=2.5$ (Dunlop et al. 2003); (7) converted from $V$ (Sandage 1973) assuming $V-R_{\mathrm{C}}=0.61$ (Fukugita et al. 1995); (8) converted from 2MASS $K_{\mathrm{s}}$ assuming $R-K_{\mathrm{s}}=2.5$ (Dunlop et al. 2003). Emission-line classifications are from Jackson \& Rawlings (1997), except for 3C 28 and 4C 12.03 which are from Willott's compilation (http://www-astro.physics.ox.ac.uk/ cjw/3crr/3crr.html), and 3C 442A which we classify as LEG based on our Palomar spectrum. The 1.4 GHz fluxes were taken from NVSS by Condon et al. (1998) and from the surveys of White \& Becker (1992) and Laing \& Peacock (1980). References for velocity dispersions: SHI90: Smith et al. (1990), H85: Heckman et al. (1985), FIF95: Fisher et al. (1995) and EFAR99: Wegner et al. (1999). References for emission-line fluxes: A: from our data, W and G: from Willott's and Grimes' compilations, respectively (http://www-astro.physics.ox.ac.uk/ cjw/3crr/3crr.html and http://www-astro.physics.ox.ac.uk/ sr/grimes.html).

between the beginning and end of the second and third night. For the first night, which started out as non-photometric, the difference was $\approx 0.7 \mathrm{mag}$. For spectrophotometry with the $4 "$ slit, we therefore waited until the middle of the first night when the conditions were more favourable. As templates for the velocity dispersion fitting we obtained spectra of one sub giant G0 star (HD 188121) and one giant K1 star (HD 28191). In order to avoid seeing-limited spectral resolution with the $1^{\prime \prime}$ slit, the template star was moved across the slit during integration.

The spectra were reduced and extracted in a standard manner using IRAF ${ }^{2}$ tasks in the twodspec and onedspec packages. Night sky lines from $\mathrm{O}$ I and from mercury in street-lamps were used to check the wavelength calibration, and an agreement of typically $\lesssim 0.5$ pixels was found, corresponding to $\lesssim 18$ and $\lesssim 32 \mathrm{~km} \mathrm{~s}^{-1}$ in the red and blue, respectively.

Two of the spectra, 3C 83.1B and 3C 386, are dominated by light from foreground stars. For this reason we do not consider these two galaxies in our analysis. In the case of $3 \mathrm{C} 83.1 \mathrm{~B}$, the star lies $3^{\prime \prime}$ to the east of the galaxy nucleus (Poulain et al. 1992; de Koff et al. 2000), and for 3C 386 the star is superposed on the optical galaxy core (Chiaberge et al. 2002).

${ }^{2}$ IRAF is distributed by the National Optical Astronomy Observatories, which are operated by the Association of Universities for Research in Astronomy, Inc., under cooperative agreement with the National Science Foundation.

\section{Template fitting procedure}

We fit stellar templates to the galaxy spectra in order to measure velocity dispersions using the direct fitting method described by Barth et al. (2002). Galaxy and stellar template spectra were extracted using aperture diameters of 1 '. 872 in order to cover the same physical scale in both spectral arms. A model spectrum, $M(x)$, where $x=\ln \lambda$ is measured in the galaxy rest frame was evaluated as

$M(x)=\{[T(x) \otimes G(x)]+C(x)\} \times P(x)$,

where $[T(x) \otimes G(x)]$ denotes the template spectrum convolved with a Gaussian of width $\sigma_{*}, C(x)$ is the continuum and $P(x)$ a polynomial. The best-fit model was found by minimizing $\chi^{2}$ using a downhill simplex method implemented in the amoeba algorithm (Press \& Spergel 1988).

We experimented with different functional forms of $C(x)$ and $P(x)$, starting with a straight line for the continuum, and a Legendre polynomial for $P(x)$. However, since the change in $x$ is small over the fitting range, coefficient degeneracies were easily introduced, hence we rescaled the fitting range to a new variable $\theta \in[0, \pi]$ and used Fourier-type expansions instead. The continuum is then expressed as

$C(\theta)=c_{0}+c_{1} \sin \theta$ 
Table 2. Table of observations. For each galaxy, the slit position angle and exposure time is shown.

\begin{tabular}{|c|c|c|c|c|c|}
\hline \multirow[b]{2}{*}{ Galaxy } & \multicolumn{2}{|c|}{$1^{\prime \prime}$ Slit } & \multicolumn{2}{|c|}{ 4" Slit } & \multirow[b]{2}{*}{ Date } \\
\hline & $\begin{array}{r}\text { PA } \\
\text { (deg) }\end{array}$ & $\begin{array}{l}T_{\exp } \\
\text { (s) }\end{array}$ & $\begin{array}{r}\text { PA } \\
\text { (deg) }\end{array}$ & $\begin{array}{r}T_{\exp } \\
\text { (s) }\end{array}$ & \\
\hline $3 \mathrm{C} 20$ & 125 & 3600 & 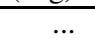 & & 11 Sep. 02 \\
\hline $3 \mathrm{C} 28$ & 59 & 2400 & $\ldots$ & $\ldots$ & 11 Sep. 02 \\
\hline 3 C 31 & 144 & 2400 & $\ldots$ & $\ldots$ & 10 Sep. 02 \\
\hline 3C 33 & 146 & 2400 & $\ldots$ & $\ldots$ & 10 Sep. 02 \\
\hline $3 \mathrm{C} 35$ & 111 & 2400 & 23 & 900 & 10 Sep. 02 \\
\hline $3 \mathrm{C} 66 \mathrm{~B}$ & 135 & 1200 & $\ldots$ & $\ldots$ & 10 Sep. 02 \\
\hline 3C 76.1 & 135 & 2400 & 130 & 900 & 12 Sep. 02 \\
\hline $3 \mathrm{C} 83.1 \mathrm{~B}^{a}$ & 166 & 1800 & 96 & 2400 & 12 Sep. 02 \\
\hline $3 \mathrm{C} 98$ & 150 & 2400 & & & 12 Sep. 02 \\
\hline 3C 388 & 71 & 2400 & 71 & 900 & 11 Sep. 02 \\
\hline $3 C 381$ & 154 & 3600 & $\ldots$ & $\ldots$ & 11 Sep. 02 \\
\hline $3 C 386^{b}$ & 12 & 2400 & 12 & 1800 & 10 Sep. 02 \\
\hline $3 \mathrm{C} 401$ & 0 & 3600 & 0 & 1200 & 11 Sep. 02 \\
\hline $3 \mathrm{C} 433$ & 66 & 2400 & $\ldots$ & $\ldots$ & 11 Sep. 02 \\
\hline $3 \mathrm{C} 442 \mathrm{~A}$ & 131 & 2400 & 57 & 900 & 12 Sep. 02 \\
\hline 3C 449 & 1 & 2400 & 8 & 900 & 10 Sep. 02 \\
\hline $3 \mathrm{C} 452$ & 0 & 2400 & & & 12 Sep. 02 \\
\hline $3 C 465$ & 30 & 1800 & 121 & 900 & 12 Sep. 02 \\
\hline 4C 11.71 & 326 & 2400 & 53 & 900 & 10 Sep. 02 \\
\hline 4C 12.03 & 17 & 2400 & 17 & 900 & 11 Sep. 02 \\
\hline 4C 35.40 & 84 & 2400 & 133 & 1200 & 12 Sep. 02 \\
\hline
\end{tabular}

a Confused with a star 3" away from the nucleus (Poulain et al. 1992; de Koff et al. 2000).

${ }^{b}$ A star is superposed on the galaxy nucleus.

and the polynomial as

$P(\theta)=p_{0}+p_{1} \cos \theta+p_{2} \cos 2 \theta+p_{3} \cos 3 \theta+p_{4} \cos 4 \theta$.

The sine and cosine terms are orthogonal, thereby breaking part of the degeneracy.

Fits to three different galaxies along with the stellar template used to construct the model are shown in Fig. 2. Generally, we found that the regions redward of $\mathrm{Ca} \mathrm{H} \& \mathrm{~K}$ and the $\mathrm{Mg} \mathrm{I} b$ absorption line complex are best suited for fitting velocity dispersions. This has also been experienced in other works, e.g. Barth et al. (2002) and Greene \& Ho (2006). We therefore tried to avoid the $\mathrm{Mg} \mathrm{I} b$ region, except for in some cases where the Fe blends redward of the region were not sufficiently covered. As Barth et al., we also find excess emission at $\approx 5200 \AA$ in several cases (caused by [N I] $5199 \AA$ emission in the host), complicating the fitting. For the red arm spectra, we therefore started several of the fits at $\approx 5220 \AA$. The results from the fitting and the fitting regions used are listed in Table 3.

The red arm spectra were easier to fit than those from the blue arm, probably because the red spectra have better signalto-noise and containing less spectral features to fit. Also, because the galaxies contain spectral features that match better with $\mathrm{K}$ stars than with $\mathrm{G}$ stars in the chosen fitting regions, the K1 template was found to produce better fits with less scatter than the G0 template. As there is also less contamination by the AGN continuum in the red part, we chose to use the red arm spectra with the K1 template. In Fig. 3 we show a comparison between measurements from the red and the blue arm in cases where spectra from both could be fitted. The errors are seen to be larger for the blue fits, and the two agree to within typically ten per cent, except for two cases, 3C 449 and 3C 98 where there are differences at the 20-30\% level.

We tested the fitting routine by constructing artificial galaxy spectra from the two stellar templates $(50 \% \mathrm{~K} 1-$ and
$50 \%$ G0-star), broadening them by a specified amount and applying the fitting routine. Noise spectra were also added, broadened to the instrumental resolution $\left(\approx 45 \mathrm{~km} \mathrm{~s}^{-1}\right)$ and scaled in order to obtain a signal-to-noise ratio of approximately 15 . The result from the simulations is shown Fig. 4, where it can be seen that the velocity dispersions are generally recovered to within $10 \%$ of the input value. As expected, at dispersions lower than the instrumental resolution there is larger scatter.

Nine of the radio galaxies in the sample have velocity dispersions from the literature, as listed in column nine of Table 1. A comparison with the literature measurements is shown in Fig. 5. There is agreement to within less than $20 \%$, except for one galaxy, 3C 388, where our estimated velocity dispersion is $36 \%$ lower than the literature value. This galaxy is one of the objects in our sample with noisier spectra, hence it has larger error bars on the velocity dispersion than the others. Part of the discrepancy could be due to our estimate and the estimate by Heckman et al. (1985) being based on different spectral regions. The blue part of the spectrum, used by Heckman et al. (1985), could be more contaminated by AGN continuum than the red part, affecting velocity dispersion estimates.

\section{Discussion}

\subsection{Conversion to AGN parameters}

We convert the velocity dispersions derived from the spectral fitting to a common physical scale following the scheme by Jørgensen et al. (1995), and thereafter to BH mass using the $M_{\mathrm{BH}}-\sigma_{*}$ relation from Tremaine et al. (2002). We find that the sample spans a relatively narrow range in $\mathrm{BH}$ mass, from $2.2 \times$ $10^{7}$ to $1.1 \times 10^{9} M_{\odot}$. Assuming that we are able to recover the velocity dispersions to within an accuracy of $\approx 10 \%$ as shown by the simulations, and including a scatter of 0.3 dex in $\log M_{\mathrm{BH}}$ (Tremaine et al. 2002) around the $M_{\mathrm{BH}}-\sigma_{*}$ relation, we estimate that we are able to determine $\mathrm{BH}$ masses to an accuracy of approximately 0.35 dex.

Narrow emission line fluxes for [O II] and [O III] were determined from our 4" slit spectra, and some were also taken from the literature, see Cols. 11 and 12 of Table 1. Under the assumption that the narrow emission lines are photoionzied by the UV continuum from the AGN, we convert to total luminosity in the narrow-line region using the relation by Rawlings \& Saunders $(1991), L_{\mathrm{NLR}} \approx 3\left(3 L_{[\mathrm{OII}]}+1.5 L_{[\mathrm{OIII}]}\right)$. In cases where only one of the two line fluxes are known (for FRIIs), we apply a relation between $L_{[\mathrm{OII}]}$ and $L_{[\mathrm{OIII}]}$ from Grimes et al. (2004), whereas for FRIs we take $L_{[\mathrm{OII}]}=L_{[\mathrm{OIII}]}$. If there is an upper limit on the flux, we take the line flux to be equal to the limit. The narrow line flux is converted to photoionizing luminosity, $Q_{\text {phot }}$, by assuming a covering factor of $\kappa=0.005$ (Willott et al. 1999) for the narrow-line gas. We estimate that the uncertainty in $\log Q_{\text {phot }}$ is rougly \pm 1 dex, which includes uncertainties related to the covering factor for the narrow-line gas and the fact that narrow lines may also be powered by shocks (Dopita \& Sutherland 1995; Inskip et al. 2002).

In order to estimate the average kinetic power transported by the jets to the lobes, we use the relation of Willott et al. (1999), $Q_{\text {jet }}=3 \times 10^{38} L_{151}^{6 / 7}$ Watts, where $L_{151}$ has units of $10^{28} \mathrm{~W} \mathrm{~Hz}^{-1} \mathrm{sr}^{-1}$. The radio luminosity at $151 \mathrm{MHz}$ for the sources in our sample was taken from Grime's compilation ${ }^{3}$. Given that Willott et al.'s relation applies to a "typical" radio

\footnotetext{
${ }^{3}$ http://www-astro.physics.ox.ac.uk/ sr/grimes.html
} 

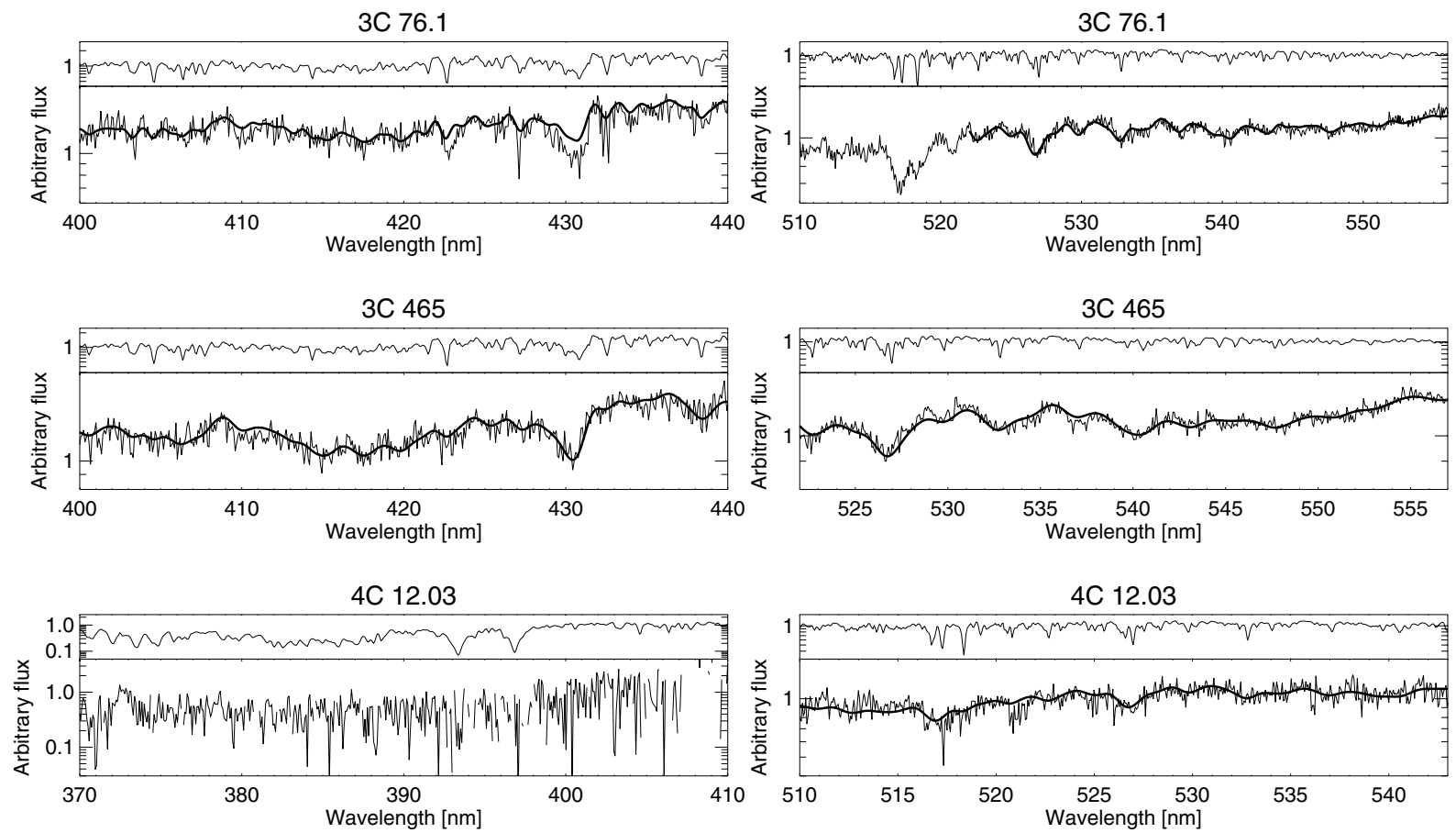

Fig. 2. Examples of radio galaxy spectra with best-fit models overplotted as solid lines where an acceptable fit could be obtained. The K1 star used as a template for the fitting is shown at the top of each panel.

Table 3. Results from the fitting. The fitting regions and signal-to-noise per pixel in the continuum is listed. Best-fit velocity dispersions normalized to the same physical aperture $\left(r_{\mathrm{ap}}=0.595 \mathrm{~h}^{-1} \mathrm{kpc}\right)$ and their confidence intervals are shown. "AGN cont." indicates that there is too much AGN contamination in the spectrum to obtain a reliable fit.

\begin{tabular}{llrlll|lllll}
\hline \hline & $\begin{array}{l}\text { Fitting region } \\
\AA\end{array}$ & $S / N$ & $\begin{array}{l}\sigma_{*} \\
\mathrm{~km} \mathrm{~s}^{-1}\end{array}$ & $\begin{array}{l}68 \% \text { CI } \\
\mathrm{km} \mathrm{s}^{-1}\end{array}$ & $\begin{array}{l}95 \% \text { CI } \\
\mathrm{km} \mathrm{s}^{-1}\end{array}$ & $\begin{array}{l}\text { Fitting region } \\
\AA\end{array}$ & $\begin{array}{l}S / N \\
\AA_{*}\end{array}$ & $\begin{array}{l}\sigma_{*} \\
\mathrm{~km} \mathrm{~s}^{-1}\end{array}$ & $\begin{array}{l}68 \% \text { CI } \\
\mathrm{km} \mathrm{s}^{-1}\end{array}$ & $\begin{array}{l}95 \% \text { CI } \\
\mathrm{km} \mathrm{s}^{-1}\end{array}$ \\
\hline 3C 20 & AGN cont. & 4 & $\ldots$ & $\ldots$ & $\ldots$ & too noisy & 1 & $\ldots$ & $\ldots$ & $\ldots$ \\
3C 28 & AGN cont. & 6 & $\ldots$ & $\ldots$ & $\ldots$ & 1 & $\ldots$ & $\ldots$ & $\ldots$ \\
3C 31 & $5220-5650$ & 25 & 247 & {$[246,251]$} & {$[244,252]$} & $4000-4500$ & 7 & 260 & {$[255,266]$} & {$[248,273]$} \\
3C 33 & $5220-5420$ & 11 & 188 & {$[178,199]$} & {$[168,210]$} & AGN cont. & 6 & $\ldots$ & $\ldots$ & $\ldots$ \\
3C 35 & $5090-5390$ & 6 & 224 & {$[215,233]$} & {$[206,243]$} & $3800-4250$ & 3 & 206 & {$[191,224]$} & {$[176,242]$} \\
3C 66B & poor fit & 18 & $\ldots$ & $\ldots$ & $\ldots$ & $4000-4470$ & 6 & 165 & {$[155,174]$} & {$[146,185]$} \\
3C 76.1 & $5220-5560$ & 18 & 193 & {$[188,198]$} & {$[183,203]$} & $4000-4470$ & 7 & 192 & {$[174,209]$} & {$[157,225]$} \\
3C 98 & $5220-5570$ & 17 & 136 & {$[128,143]$} & {$[120,152]$} & $4000-4420$ & 7 & 159 & {$[149,170]$} & {$[140,181]$} \\
3C 388 & $5150-5740$ & 15 & 232 & {$[211,243]$} & {$[195,259]$} & too noisy & 2 & $\ldots$ & $\ldots$ & $\ldots$ \\
3C 381 & $5100-5410$ & 9 & 221 & {$[212,231]$} & {$[202,241]$} & too noisy & 1 & $\ldots$ & $\ldots$ & $\ldots$ \\
3C 401 & $5100-5230$ & 5 & 136 & {$[95,166]$} & {$[65,284]$} & too noisy & 1 & $\ldots$ & $\ldots$ & $\ldots$ \\
3C 433 & $5220-5700$ & 10 & 128 & {$[121,131]$} & {$[115,136]$} & too noisy & 1 & $\ldots$ & $\ldots$ & $\ldots$ \\
3C 442A & $5100-5590$ & 15 & 198 & {$[188,208]$} & {$[193,203]$} & $4000-4450$ & 6 & 216 & {$[207,225]$} & {$[198,234]$} \\
3C 449 & $5100-5650$ & 17 & 263 & {$[258,267]$} & {$[254,271]$} & $3800-4500$ & 6 & 192 & {$[187,198]$} & {$[181,204]$} \\
3C 452 & $5120-5310$ & 7 & 289 & {$[284,296]$} & {$[274,307]$} & AGN cont. & 4 & $\ldots$ & $\ldots$ & $\ldots$ \\
3C 465 & $5220-5570$ & 19 & 338 & {$[332,344]$} & {$[326,350]$} & $4000-4400$ & 7 & 362 & {$[351,375]$} & {$[340,388]$} \\
4C 11.71 & $5220-5600$ & 8 & 227 & {$[224,232]$} & {$[220,234]$} & $4000-4450$ & 6 & 250 & {$[242,259]$} & {$[234,267]$} \\
4C 12.03 & $5100-5420$ & 8 & 245 & {$[231,257]$} & {$[219,271]$} & too noisy & 1 & $\ldots$ & $\ldots$ & $\ldots$ \\
4C 35.40 & $5220-5570$ & 16 & 205 & {$[198,205]$} & {$[190,213]$} & $4000-4420$ & 6 & 193 & {$[184,200]$} & {$[176,209]$} \\
\hline
\end{tabular}

${ }^{a}$ Despite good signal-to-noise in the red part, for unknown reasons, an acceptable fit could not be obtained.

galaxy of median age, \pm 1 dex is a reasonable estimate for the uncertainty in $\log Q_{\text {jet }}$.

We also derive the dimensionless ratio between the jet luminosity and the photoionizing luminosity, $\log \left(Q_{\text {jet }} / Q_{\text {phot }}\right)$, and estimate an uncertainty in this parameter of approximately \pm 1.5 dex. The $Q_{\text {jet }} / Q_{\text {phot }}$ ratio can be seen as the ratio of kinetic to radiative energy output by the AGN, and indicates how effectively jets are formed. A small ratio implies that the energy output is dominated by thermal radiation from an accretion disk, whereas a large ratio indicates that most of the energy goes into forming jets, i.e. to kinetic energy, and that the accretion process thus may be radiatively inefficient.

\subsection{The Ledlow-Owen relation}

The parameters $Q_{\text {jet }}, Q_{\text {phot }}$ and their ratio are plotted as a function of BH mass in Fig. 6. The line of separation between FRIs and FRIIs, $L_{\text {radio }} \propto L_{\text {optical }}^{1.8}($ Ledlow \& Owen 1996), can be written as

$\log \left(\frac{P_{1.4}}{\mathrm{WHz}^{-1}}\right)=-0.67 M_{R}+10.13$ 


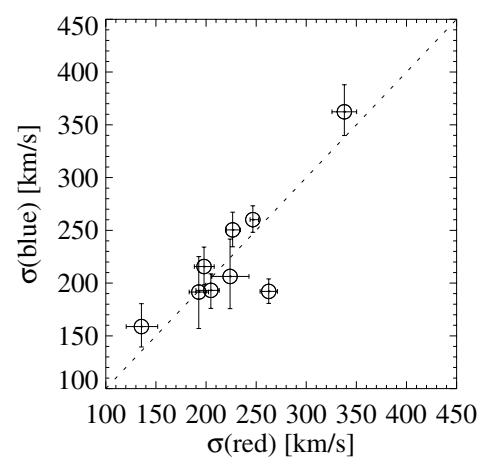

Fig. 3. Comparison of red and blue velocity dispersions. Error bars represent $95 \%$ confidence intervals.

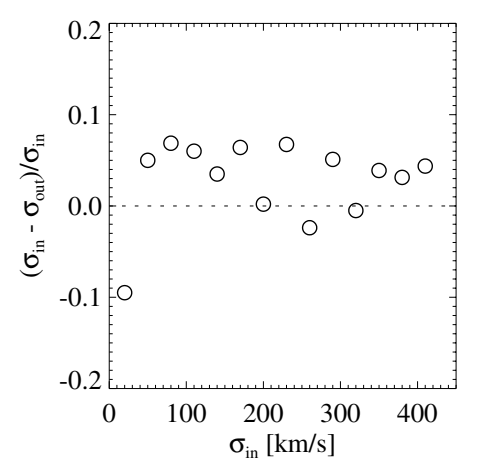

Fig. 4. Results from simulations with artificial galaxy spectra. The input velocity dispersions, $\sigma_{\text {in }}$, are recovered $\left(\sigma_{\text {out }}\right)$ to within $10 \%$.

where $M_{R}$ is the optical absolute magnitude of the host and $P_{1.4}$ is the radio power at $1.4 \mathrm{GHz}$. By using the relation by McLure \& Dunlop (2001) which relates $M_{R}$ to $M_{\mathrm{BH}}$ and the relation from Willott et al. (1999) linking $P_{1.4}$ to $Q_{\text {jet }}$, we obtain

$\left(\frac{Q_{\mathrm{jet}}}{\mathrm{W}}\right)=6.17 \times 10^{27}\left(\frac{M_{\mathrm{BH}}}{M_{\odot}}\right)^{0.92}$.

This translates into $Q_{\text {jet }} \simeq 5 \times 10^{-4} L_{\text {Edd }}$, implying that the FRI/FRII transition occurs at a ratio of $\simeq 0.0005$ between jet power and Eddington luminosity if $M_{\mathrm{BH}} \approx 10^{8} M_{\odot}$. Ghisellini $\&$ Celotti (2001) derive a ratio of 0.015 , but this is probably erroneous ${ }^{4}$. The left-hand panel of Fig. 6 shows $Q_{\text {jet }}$ as a function of $M_{\mathrm{BH}}$, and the Ledlow-Owen relation in the form $Q_{\text {jet }}=5 \times$ $10^{-4} M_{\mathrm{BH}}$ is indicated with a solid line and seems to fit the data well.

The Ledlow-Owen relation can also be written in terms of photoionizing luminosity through the radio-optical correlation for radio galaxies (Willott et al. 1999). Doing this, we find $Q_{\text {phot }} \simeq 0.0037 L_{\mathrm{Edd}}$ for the typical $\mathrm{BH}$ mass in our sample. (Ghisellini \& Celotti 2001 derive $Q_{\text {phot }} \simeq 0.005 L_{\text {Edd }}$ ). The solid line in the middle panel of Fig. 6 shows the Ledlow-Owen relation recast in the two variables $Q_{\text {phot }}$ and $M_{\mathrm{BH}}$ and can be seen to fit the transition region quite well. However, the separation of FRIs and FRIIs appears to be cleaner in the diagram of $\log Q_{\text {phot }}$ vs. $\log M_{\mathrm{BH}}$, probably because $Q_{\text {jet }}$ is more influenced by external factors, such as conditions in the interstellar medium of the host. If the accretion efficiency is approximately $10 \%$, and assumed to be in the form of a standard optically thick, geometrically thin accretion disk (Shakura \& Sunyaev 1973), the relation

\footnotetext{
${ }^{4}$ The factor in Eq. (2) of Ghisellini \& Celotti (2001) should be $3 \times$ $10^{15}$ instead of $3 \times 10^{21}$.
}

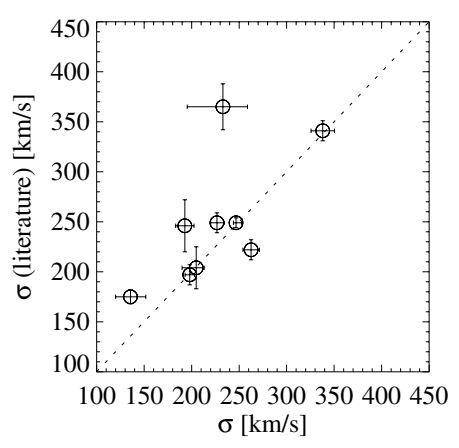

Fig. 5. Comparison with dispersions from the literature.

$Q_{\text {phot }} \approx 0.004 L_{\text {Edd }}$ indicates that the FRI/FRII transition occurs at $\sim 4 \%$ of the Eddington rate.

The transition can also be described as occurring at an almost fixed ratio between $Q_{\text {jet }}$ and $Q_{\text {phot }}$, i.e. at a fixed "radio loudness". Using the relations above, we find $Q_{\text {jet }} / Q_{\text {phot }} \approx 0.1$, shown as a solid line in the rightmost panel of Fig. 6, but the sources do not separate as well in this diagram as in the other two.

Both AGN and X-ray binaries (XRBs) are associated with accretion onto compact objects and the production of jets. The two have therefore been compared at several occations (e.g. Merloni et al. 2003; Falcke et al. 2004; Maccarone 2003; McHardy et al. 2006). XRBs are characterized by three different spectral states (see e.g. Gallo et al. 2003). The low/hard state has a hard power-law spectrum, a weak thermal component from an accretion disk/flow, and steady jet emission is seen at radio wavelengths. FRIs have been suggested as the high BH mass analogue of the low/hard state in XRBs (see e.g. Merloni et al. 2003; Falcke et al. 2004) and having accretion flows with low accretion rates and inefficient cooling, such as advection-dominated accretion flows (Rees et al. 1982; Narayan \& Yi 1994). FRIIs, on the other hand, are thought of as analogous to the high/soft and very high state XRBs with accretion disks modeled as standard, optically thick, geometrically thin disks (Shakura \& Sunyaev 1973). XRBs in the high/soft state show no strong or steady radio emission, and jet formation is thought to be inhibited. In the so-called very high state, thought to occur at high accretion rates, XRBs show transient jet phenomena.

If, when we derive the relations in Eqs. (4) and (5), keep the BH mass dependence instead of substituting the mean black hole mass of the sample, we find that $Q_{\mathrm{phot}} / L_{\mathrm{Edd}} \propto\left(M_{\mathrm{BH}} / M_{\odot}\right)^{-0.14}$ and $Q_{\text {jet }} / L_{\mathrm{Edd}} \propto\left(M_{\mathrm{BH}} / M_{\odot}\right)^{-0.08}$. Hence both the photoionizing and jet luminosity in Eddington units at the FR/FRII transition is weakly dependent on BH mass. It is too weak to be noticeable in a sample of radio galaxies, but when extrapolated to XRBs there should be roughly a factor of 10 difference. From the Ledlow-Owen relation we therefore expect that the FRI/FRII transition luminosity in Eddington units is about a factor of 10 lower than for spectral state transition in XRBs. This does indeed seem to be observed. For the FRI/FRII transition we observe transition luminosities of $\approx 0.4 \%$ of the Eddington luminosity, see Fig. 6. The transition luminosity of XRBs from the high/soft to the low/hard state is $\approx 2 \%$ of the Eddington luminosity (may be up to four times higher for the low/hard to high/soft transition) (Maccarone et al. 2003). The concurrence of observed transition luminosities with those predicted from radio galaxy scaling relations may indicate that the transition between low/hard and high/soft states in XRBs is indeed similar in nature to the FRI/FRII transition. Maccarone (2003) discusses that 

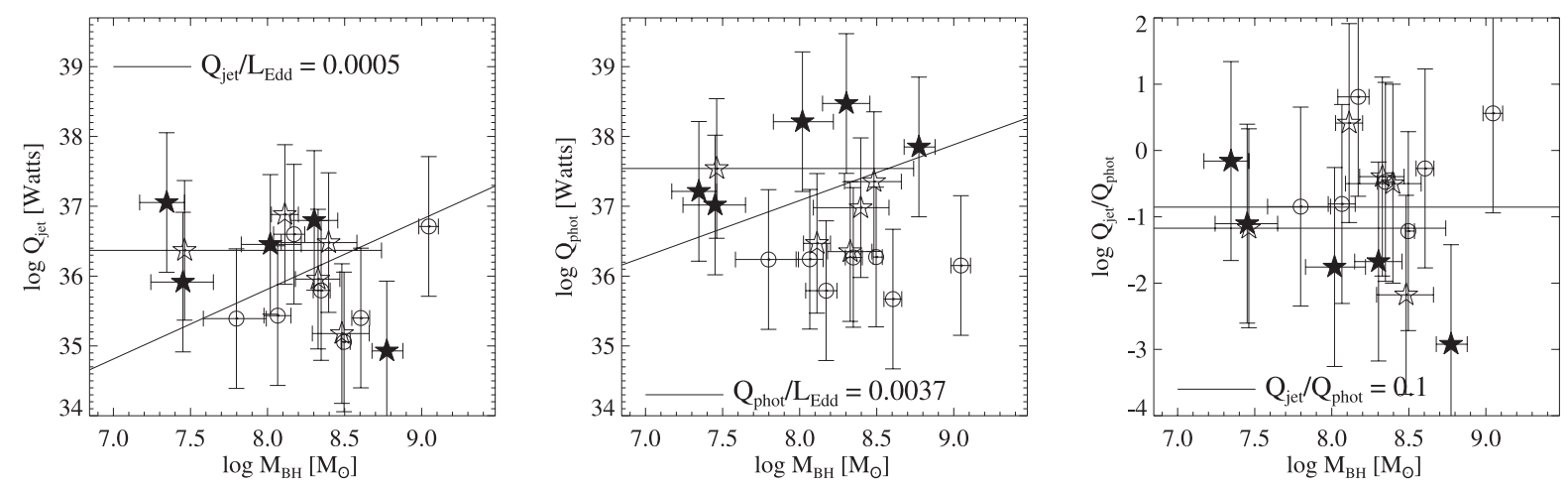

Fig. 6. From left to right, jet power, photoionizing luminosity and their ratio as a function of BH mass. An uncertainty of \pm 1 dex in $Q_{\text {jet }}$ and $Q_{\text {phot }}$ has been assumed, and the $95 \%$ confidence interval on BH mass is used. Symbols are as in Fig. 1.

neutron star XRBs may have higher transition luminosities than BH XRBs, also indicating that the transition luminosity may depend on the mass of the compact object.

If FRIIs are analogues to the very high state XRBs with thermally unstable and radiation pressure dominated accretion disks (Meier 2001), the FRI/FRII transition could correspond to a switch between the low/hard and the very high state, without going through the high/soft state. Meier (2001) offers a solution which has the transition luminosity relative to the Eddington luminosity proportional to $M_{\mathrm{BH}}^{-1 / 8}$ (see also Chen et al. 1995; Merloni 2003). There is some inconsistency in the implied transition luminosities in this scenario because the very high state in XRBs is thought to occur at a (bolometric) luminosity of 20$30 \%$ of the Eddington luminosity (Maccarone et al. 2003; Meier 2001), whereas we find $<1 \%$ for the radio galaxies. The discrepancy could be due to our different methods of estimating the bolometric/photoionizing luminosity, but another possibility is that the physical conditions (e.g. density) in the accretion flow close to the $\mathrm{BH}$ are different for radio galaxies and XRBs.

Sources that do not follow the Ledlow-Owen relation may be the ones in clusters or high density environments (Marchesini et al. 2004). Besides external density in this case probably being a dominant factor for the radio morphology, another possible explanation is that mergers or collisions in cluster galaxies may have altered the spin of the BH (Natarajan \& Pringle 1998) and $\mathrm{BH}$ spin is thought to be important for the formation of jets.

The Ledlow-Owen relation nevertheless seems to be an indication that the FRI/FRII morphology is largely determined by nuclear AGN parameters. In hindsight it is perhaps not surprising that the empirical scaling relations used by Bicknell (1995) to explain the Ledlow-Owen relation relate to the central stellar velocity dispersion.

\section{Summary and conclusions}

We have investigated $\mathrm{BH}$ masses and narrow emission line luminosities in a sample of FRI and FRII radio galaxies. BH masses were estimated via the $M_{\mathrm{BH}}-\sigma_{*}$ relation by determining central stellar velocity dispersions from medium resolution spectra, and photoionizing luminosities were estimated from spectrophotometry of the narrow [O II $] 3727 \AA$ and [O III]5007 $\AA$ emission lines. The measured velocity dispersions span the range $130-340 \mathrm{~km} \mathrm{~s}^{-1}$ with a mean of $\approx 215 \mathrm{~km} \mathrm{~s}^{-1}$. Including the scatter in the $M_{\mathrm{BH}^{-}} \sigma_{*}$ relation, we estimate that we are able to determine $\mathrm{BH}$ masses with an accuracy of $\approx 0.35$ dex. We find FRIs and FRIIs to have the similar $\mathrm{BH}$ mass distributions, confirming previous results (e.g. McLure et al. 2004; Marchesini et al. 2004).

The data fit the Ledlow-Owen relation well when it is rewritten in terms of jet luminosity, photoionizing luminosity and BH mass (Ghisellini \& Celotti 2001). The separation of FRIs and FRIIs appears cleaner in the $Q_{\mathrm{phot}}-M_{\mathrm{BH}}$ plane than in the $Q_{\text {jet }}-M_{\mathrm{BH}}$ plane, indicating that FRIs and FRIIs are more easily separated in terms of nuclear parameters than in terms of parameters such as $Q_{\text {jet }}$ which may be more influenced by external factors. However, it is still possible that the FR/FRII transition may be caused by a mixture of both nuclear and external factors.

We discuss whether the FRI/FRII transition can be compared to spectral state transitions in XRBs, and highlight the fact that the Ledlow-Owen relation predicts a $\mathrm{BH}$ mass dependence in the transition luminosity in Eddington units. This implies that radio galaxies change from FRI to FRII at luminosities (in Eddington units) that are a factor of 10 lower that those of XRBs.

Acknowledgements. The authours thank the referee for comments which helped to improve the original manuscript. This research has made use of the NASA/IPAC Extragalactic Database (NED) which is operated by the Jet Propulsion Laboratory, California Institute of Technology, under contract with the National Aeronautics and Space Administration. The authors would also like to thank the maintainers of the DRAGN web page (http://www.jb.man.ac.uk/altas) which was used in this work.

\section{References}

Barth, A. J., Ho, L. C., \& Sargent, W. L. W. 2002, AJ, 124, 2607 Baum, S. A., Zirbel, E. L., \& O’Dea, C. P. 1995, ApJ, 451, 88 Bicknell, G. V. 1995, ApJS, 101, 29

Cao, X., \& Rawlings, S. 2004, MNRAS, 349, 1419

Chen, X., Abramowicz, M. A., Lasota, J.-P., Narayan, R., \& Yi, I. 1995, ApJ, 443, L61

Chiaberge, M., Capetti, A., \& Celotti, A. 2002, A\&A, 394, 791

Comins, N. F., \& Owen, F. N. 1991, ApJ, 382, 108

Condon, J. J., Cotton, W. D., Greisen, E. W., et al. 1998, AJ, 115, 1693

de Koff, S., Best, P., Baum, S. A., et al. 2000, ApJS, 129, 33

De Young, D. S. 1993, ApJ, 405, L13

Dopita, M. A., \& Sutherland, R. S. 1995, ApJ, 455, 468

Dunlop, J. S., McLure, R. J., Kukula, M. J., et al. 2003, MNRAS, 340, 1095

Fabbiano, G., Trinchieri, G., Elvis, M., Miller, L., \& Longair, M. 1984, ApJ, 277, 115

Falcke, H., Körding, E., \& Markoff, S. 2004, A\&A, 414, 895

Fanaroff, B. L., \& Riley, J. M. 1974, MNRAS, 167, 31P

Fisher, D., Illingworth, G., \& Franx, M. 1995, ApJ, 438, 539

Fukugita, M., Shimasaku, K., \& Ichikawa, T. 1995, PASP, 107, 945

Gallo, E., Fender, R. P., \& Pooley, G. G. 2003, MNRAS, 344, 60

Ghisellini, G., \& Celotti, A. 2001, A\&A, 379, L1

Gopal-Krishna, \& Wiita, P. J. 2000, A\&A, 363, 507

Gopal-Krishna, \& Wiita, P. J. 2001, A\&A, 373, 100

Greene, J. E., \& Ho, L. C. 2006, ApJ, 641, 117 
Grimes, J. A., Rawlings, S., \& Willott, C. J. 2004, MNRAS, 349, 503 Heckman, T. M., Illingworth, G. D., Miley, G. K., \& van Breugel, W. J. M. 1985, ApJ, 299, 41

Inskip, K. J., Best, P. N., Rawlings, S., et al. 2002, MNRAS, 337, 1381

Jackson, C. A., \& Wall, J. V. 1999, MNRAS, 304, 160

Jackson, N., \& Rawlings, S. 1997, MNRAS, 286, 241

Jørgensen, I., Franx, M., \& Kjaergaard, P. 1995, MNRAS, 276, 1341

Kaiser, C. R., \& Alexander, P. 1997, MNRAS, 286, 215

Kormendy, J., \& Richstone, D. 1995, ARA\&A, 33, 581

Laing, R. A. 1994, in The Physics of Active Galaxies, ed. G. V. Bicknell, M. A. Dopita, \& P. J. Quinn, ASP Conf. Ser., 54, 227

Laing, R. A. 1996, in Energy Transport in Radio Galaxies and Quasars, ASP Conf. Ser., 100, 241

Laing, R. A., \& Peacock, J. A. 1980, MNRAS, 190, 903

Laing, R. A., Riley, J. M., \& Longair, M. S. 1983, MNRAS, 204, 151

Laing, R. A., Jenkins, C. R., Wall, J. V., \& Unger, S. W. 1994, in The Physics of Active Galaxies, ASP Conf. Ser., 54, 201

Lebofsky, M. J. 1981, ApJ, 245, L59

Ledlow, M. J., \& Owen, F. N. 1996, AJ, 112, 9

Lilly, S. J., \& Longair, M. S. 1984, MNRAS, 211, 833

Lilly, S. J., Longair, M. S., \& Miller, L. 1985, MNRAS, 214, 109

Maccarone, T. J. 2003, A\&A, 409, 697

Maccarone, T. J., Gallo, E., \& Fender, R. 2003, MNRAS, 345, L19

Magorrian, J., Tremaine, S., Richstone, D., et al. 1998, AJ, 115, 2285

Marchesini, D., Celotti, A., \& Ferrarese, L. 2004, MNRAS, 351, 733

Martel, A. R., Baum, S. A., Sparks, W. B., et al. 1999, ApJS, 122, 81

McHardy, I. M., Koerding, E., Knigge, C., Uttley, P., \& Fender, R. P. 2006, Nature, 444, 730

McLure, R. J., \& Dunlop, J. S. 2001, MNRAS, 327, 199

McLure, R. J., Willott, C. J., Jarvis, M. J., et al. 2004, MNRAS, 351, 347
Meier, D. L. 1999, ApJ, 522, 753

Meier, D. L. 2001, ApJ, 548, L9

Merloni, A. 2003, MNRAS, 341, 1051

Merloni, A., Heinz, S., \& di Matteo, T. 2003, MNRAS, 345, 1057

Narayan, R., \& Yi, I. 1994, ApJ, 428, L13

Natarajan, P., \& Pringle, J. E. 1998, ApJ, 506, L97

Oke, J. B., \& Gunn, J. E. 1982, PASP, 94, 586

Owen, F. N., \& Laing, R. A. 1989, MNRAS, 238, 357

Owen, F. N., \& Ledlow, M. J. 1994, in The Physics of Active Galaxies, ed. G. V. Bicknell, M. A. Dopita, \& P. J. Quinn, ASP Conf. Ser., 54, 319

Owen, F. N., \& White, R. A. 1991, MNRAS, 249, 164

Poulain, P., Nieto, J.-L., \& Davoust, E. 1992, A\&AS, 95, 129

Press, W. H., \& Spergel, D. N. 1988, ApJ, 325, 715

Rawlings, S., \& Saunders, R. 1991, Nature, 349, 138

Rees, M. J., Phinney, E. S., Begelman, M. C., \& Blandford, R. D. 1982, Nature, 295, 17

Reynolds, C. S., di Matteo, T., Fabian, A. C., Hwang, U., \& Canizares, C. R. 1996, MNRAS, 283, L111

Sandage, A. 1973, ApJ, 183, 711

Saunders, R., Baldwin, J. E., Rawlings, S., Warner, P. J., \& Miller, L. 1989, MNRAS, 238, 777

Shakura, N. I., \& Sunyaev, R. A. 1973, A\&A, 24, 337

Smith, E. P., \& Heckman, T. M. 1989, ApJS, 69, 365

Smith, E. P., Heckman, T. M., \& Illingworth, G. D. 1990, ApJ, 356, 399

Tremaine, S., Gebhardt, K., Bender, R., et al. 2002, ApJ, 574, 740

Wegner, G., Colless, M., Saglia, R. P., et al. 1999, MNRAS, 305, 259

White, R. L., \& Becker, R. H. 1992, ApJS, 79, 331

Willott, C. J., Rawlings, S., Blundell, K. M., \& Lacy, M. 1999, MNRAS, 309, 1017

Yates, M. G., \& Longair, M. S. 1989, MNRAS, 241, 29 